Revue internationale P.M.E.

Économie et gestion de la petite et moyenne entreprise

\title{
Le renouvellement du tissu productif à travers la création d'entreprises en France
}

\section{Jean Bonnet}

Volume 11, numéro 1, 1998

URI : https://id.erudit.org/iderudit/1009034ar

DOI : https://doi.org/10.7202/1009034ar

Aller au sommaire du numéro

Éditeur(s)

Presses de l’Université du Québec

ISSN

0776-5436 (imprimé)

1918-9699 (numérique)

Découvrir la revue

Citer cet article

Bonnet, J. (1998). Le renouvellement du tissu productif à travers la création d'entreprises en France. Revue internationale P.M.E., 11(1), 9-40.

https://doi.org/10.7202/1009034ar
Résumé de l'article

L'importance des créations d'entreprises en France depuis la fin des années 1970 résulte de la conjonction d'occasions de créations d'entreprises dans les secteurs industriels et tertiaires et d'une offre d'entrepreneurs importante. La diminution du coût d'opportunité d'entreprendre contribue au renouveau entrepreneurial puisque environ $50 \%$ des créateurs d'entreprises sont d'anciens chômeurs. La forte reproduction sociale dans la création d'entreprises conjuguée au fait précédent nous permet d'entrevoir la diversité des motivations et des conditions de la création. Cette diversité de la création d'entreprises se retrouve à l'échelle sectorielle et à l'échelle spatiale. Les aides (financières, de conseil) contribuent à la pérennité des nouvelles entreprises. Cependant, une réflexion plus approfondie devrait être menée dans une problématique de démographie d'entreprises puisque les aides au maintien du tissu productif sont aussi une solution de rechange à la création d'entreprises par les chômeurs. 


\title{
Le renouvellement du tissu productif à travers la création d'entreprises en France
}

Jean BONNET

Université de Caen

\section{MOTS CLÉS}

\section{Création d'entreprises - Occasions de créer \\ Coût d'opportunité d'entreprendre - Reproduction sociale Culture entrepreneuriale - Aides à la création d'entreprises}

\begin{abstract}
RESUMÉ
L'importance des créations d'entreprises en France depuis la fin des années 1970 résulte de la conjonction d'occasions de créations d'entreprises dans les secteurs industriels et tertiaires et d'une offre d'entrepreneurs importante. La diminution du coût d'opportunité d'entreprendre contribue au renouveau entrepreneurial puisque environ $50 \%$ des créateurs d'entreprises sont d'anciens chômeurs. La forte reproduction sociale dans la création d'entreprises conjuguée au fait précédent nous permet d'entrevoir la diversité des motivations et des conditions de la création. Cette diversité de la création d'entreprises se retrouve à l'échelle sectorielle et à l'échelle spatiale. Les aides (financières, de conseil) contribuent à la pérennité des nouvelles entreprises. Cependant, une réflexion plus approfondie devrait être menée dans une problématique de démographie d'entreprises puisque les aides au maintien du tissu productif sont aussi une solution de rechange à la création d'entreprises par les chômeurs.
\end{abstract}

\section{L'AUTEUR}

Jean Bonnet est maître de conférences à la Faculté de sciences économiques et de gestion de Caen. Ses recherches portent sur l'entrepreneuriat et la mesure du renouvellement du tissu productif en intégrant la dimension régionale et locale.

Adresse : LERE, Faculté de sciences économiques et de gestion, Université de Caen, esplanade de la Paix, 14032 Caen cedex. Courriel : bonnet@econ.unicaen.fr 


\begin{abstract}
The growth in the number of start-ups in France since the end of the 1970's results both from the possibilities to start up an enterprise in the industrial and service sectors and from the great supply of entrepreneurs. The reduction in the opportunity cost of entrepreneurship has contributed to the renewal of entrepreneurship since approximately $50 \%$ of entrepreneurs were formerly unemployed. The strong social reproduction in start-ups combined with this reduction enable us to see the diversity of motivations and conditions that accompany start-ups. This diversity is also sectorial and spatial. Financial aid and counselling contribute to the perreniality of new enterprises. However, this study should be taken one step further. Enterprise demography shows that the financial and legal aid given to firms in order to keep them in activity constitute an alternative to start-ups created by the unemployed.
\end{abstract}

\title{
RESUMEN
}

La importancia de la creacion de empresas en Francia desde finales de 1970 es el resultado de la conjuncion de las oportunidades de creacion de empresas en los sectores industriales y terciarios y de una oferta importante de empresarios. La disminucion del coste de oportunidad de emprender contribuye a la recuperacion empresarial ya que alrededor del $50 \%$ de los creadores de empresas son antiguos parados. La fuerte reproduccion social en la creacion de empresas conjugada con el hecho precedente nos permite entrever la diversidad de motivos y condiciones de la creacion. Esta diversidad de creacion de empresas se encuentra en la escala sectorial y espacial. Las ayudas (financieras, de consejo) contribuyen a la perennidad de las nuevas empresas. Sin embargo, deberia de llevarse a cabo una reflexion mas profunda de una problematica de demografia de empresas puesto que las ayudas para el mantenimiento del tejido productivo son tambien una alternativa a la creacion de empresas para los parados.

\section{Introduction}

L'importance de la transmission des entreprises et des flux d'entreprises (créations et disparitions d'entreprises) depuis quelques années a suscité de nombreuses études sur le renouvellement du tissu productif en France (Bonneau et Francoz, 1995 ; Francoz et Bonneau, 1994). L'Institut national des statistiques et des études économiques (INSEE) estime que le tiers environ des entreprises toujours existantes au début de 1995 ont été créées ou reprises lors des cinq années précédentes. Elles emploient environ $20 \%$ des effectifs totaux du secteur privé (salariés et chefs d'entreprises). Les flux de créations d'entreprises représentent une part importante du stock des entreprises puisque, toutes tailles confondues, le taux de création d'entreprises ex nihilo était de 7,6\% en 1987 par rapport au parc d'entreprises repris dans la source du «Système informatique de répertoire des entreprises et des établissements » (SIRENE). En ajoutant les reprises, on obtient un taux de renouvellement brut de $9,9 \%$. Les défaillances et surtout les cessations volontaires 
d'activités réduisent fortement le solde net, mais le parc d'entreprises s'accroît de façon significative, environ $2 \%$ par an en moyenne ${ }^{1}$.

Le renouveau entrepreneurial de la période récente résulte de la conjonction d'opportunités de créations d'entreprises dans les secteurs industriels et tertiaires et d'une offre d'entrepreneurs importante. Il exerce une influence importante (souvent sous-estimée par la théorie économique sur le renouvellement des entreprises) sur la création des emplois et sur la diffusion de nouvelles techniques et de nouvelles formes d'organisation du travail.

$\mathrm{Si}$ on s'attache aux caractéristiques socio-économiques des créateurs d'entreprises, on constate non seulement une très forte reproduction sociale des créateurs d'entreprises (Kombou, 1986 ; Kombou et Kochanski, 1988; Gollec et Lauhle, 1987, Bonneau et Thirion, 1997), mais aussi une forte proportion de chômeurs créateurs d'entreprises pour lesquels le coût d'opportunité d'entreprendre est faible. Cette forte reproduction sociale et cette culture entrepreneuriale se retrouvent à l'échelle spatiale, les conditions permissives de l'augmentation du nombre des entreprises dans la période récente favorisant certains territoires (Bonnet, 1997). La liaison entre chômage et création d'entreprises à l'échelle spatiale est plus complexe puisqu'elle fait intervenir les migrations, le dynamisme de certains territoires et la modification des facteurs de localisation des nouvelles activités motrices.

L'action des pouvoirs publics en faveur de la création d'entreprises, sur la prévention des défaillances ou sur les transmissions d'entreprises, a des répercussions indéniables sur le renouvellement du tissu productif. Cependant, ces différentes mesures peuvent s'avérer contradictoires et annihiler leurs effets. Ainsi, une politique de maintien des entreprises en place peut s'interpréter comme une politique substituable à la création d'entreprises. Il existe alors un conflit à vouloir favoriser la création d'entreprises et en même temps préserver à tout prix les entreprises de la faillite.

Le législateur a, en fait, par ses lois sur la préservation du tissu productif (lois sur la défaillance de 1984-1985) rendu paradoxalement plus difficile la création de nouvelles entreprises, les banques étant réticentes à prêter aux créateurs de nouvelles entreprises. Une modification de la Loi sur les défaillances est intervenue en 1994. Elle redonne aux créanciers certaines garanties dans la mesure où la liquidation de biens aura lieu plus tôt pour un nombre appréciable d'entreprises défaillantes, ce qui limite bien souvent l'aggravation de la situation de l'entreprise que l'on a constaté dans la période d'observation de six mois prévue par les lois de 1984-1985.

1. Annexe I. 


\section{Le renouveau entrepreneurial des années $\mathbf{1 9 8 0}$}

La création d'entreprise est la rencontre d'un individu et d'un projet économique, la condition permissive de la création d'entreprises est que le gain escompté du projet excède son coût d'opportunité. Certaines périodes conjoncturelles, certaines évolutions structurelles ou institutionnelles vont modifier soit le coût d'opportunité d'entreprendre et par là l'offre d'entrepreneurs, soit le nombre de projets rentables.

Dans le cas de la France, on peut recenser trois périodes de forte intensité entrepreneuriale depuis les années 1930 :

- la période de l'après-crise de 1929 , où la diminution très forte du coût d'opportunité d'entreprendre (chômage important), a entraîné la création de nombreuses petites entreprises artisanales ${ }^{2}$,

- la période de forte croissance de la reconstruction et des années 1950, où la croissance de la demande et les perspectives de profit ont entraîné de nombreuses créations d'entreprises,

- enfin, la période plus récente de la fin des années 1970 jusqu'à nos jours, qui combine des occasions de créations d'entreprises ${ }^{3}$ et une offre d'entrepreneurs importante suscitée par un chômage élevé et une nouvelle mentalité favorable à l'entrepreneuriat.

\section{Des occasions de créations dans les secteurs tertiaires}

Les occasions de créations d'entreprises sont nombreuses :

- dans les secteurs des services aux ménages où les nombreux besoins de soins, d'assistance, d'accompagnement pour les personnes âgées et la modification du comportement de la femme par rapport au travail nécessitent le recours à des services ménagers marchands autrefois effectués dans la cellule familiale. L'augmentation du niveau de vie permet, par ailleurs, l'accès à des services dits «supérieurs ». Les services offrant de nombreuses occasions d'entreprises sont les services de la restauration

2. Les données de la source de l'Union nationale des entreprises dans l'industrie et le commerce (UNEDIC) montrent alors une rupture dans la concentration de la part de l'emploi dans les grands établissements industriels entre 1932 et 1937 (Didier, 1982).

3. La crise de 1973 a entraîné, de la part des grandes entreprises, des stratégies de recentrage sur leurs activités principales avec l'externalisation d'un certain nombre de services banals et spécialisés, des stratégies d'essaimage dans la mesure où en se désengageant d'un certain nombre de territoires, les grandes entreprises ont favorisé la reconversion de leurs salariés en les aidant à créer leur entreprise. 
rapide, de soins à domicile, les services liés au tourisme et aux équipements touristiques, les services récréatifs, culturels ou sportifs ;

- dans les secteurs des services aux entreprises où la complexification de l'activité économique, les liaisons plus importantes et plus diverses de l'entreprise avec son environnement nécessitent le recours à des services de spécialité. La recherche de la diminution des coûts dans les grandes entreprises s'est traduite, par ailleurs, par la sous-traitance de services « logistiques ». Les services offrant de nombreuses occasions de créations d'entreprises sont alors les services d'études informatiques et d'organisation, de conseil en information et documentation, les services des régies publicitaires, les services juridiques et les services divers (nettoyage, gardiennage).

\section{Des occasions de créations dans les secteurs industriels}

Un certain nombre de conditions permissives ont amené l'augmentation du nombre des entreprises industrielles eu égard à la tendance lourde de concentration du tissu productif dans la longue période.

Les raisons invoquées pour expliquer la meilleure résistance des petites et moyennes entreprises industrielles ${ }^{4}$ tiennent d'abord à leur moindre dépendance énergétique due à leur moindre capitalisation, ensuite, à leur statut d'entreprises « abritées » à cause de leur plus faible ouverture sur les marchés étrangers, et, enfin, à leur plus grande flexibilité dans la gestion de la main-d'œuvre liée à leur position d'entreprises sous-traitantes ayant déjà intégré l'incertitude dans leur fonction de production (Delattre, 1982 ; Devilliers, 1987 ; De Bandt, 1988). À ces raisons, que l'on pourrait qualifier de «négatives » - elles se comportent relativement mieux parce que les autres font moins bien -, on doit ajouter des qualités de souplesse (par rapport à l'organisation du travail, à la circulation de l'information et au type de management) et de réactivité (par rapport à l'évolution de la conjoncture - recherche de nouveaux marchés -, par rapport au changement technique - imitation). Les conditions internes du succès des PMI sont alors « le style de management, le choix des objectifs, la dimension stratégique assurée, l'organisation et l'animation de l'activité, bref le système de gestion » (Marchesnay, 1984).

4. Une petite et moyenne entreprise industrielle (PMEI ou PMI) est une entreprise employant entre 20 et 499 salariés - on distingue les grandes PMI (entre 200 et 499 salariés) -, les petites entreprises industrielles (PEI) emploient moins de 20 salariés, les très petites entreprises industrielles (TPEI) emploient moins de 10 salariées, les grandes entreprises industrielles (GEI) plus de 500. 
L'apparition d'une demande de diversité a permis la naissance et le développement de «niches de marché» favorables aux petites entreprises ${ }^{5}$. Les secteurs de l'imprimerie, presse et édition, les produits de la transformation des matières plastiques et certains secteurs liés à l'habillement ont constitué ces niches de marché. Parallèlement à un désir de consommation de biens finals de plus en plus diversifiés, spéciaux ou de qualité, il existe aussi une demande de consommation intermédiaire de demi-produits de plus en plus élaborés. «Un produit est le fruit d'une chaîne technologique de plus en plus longue ${ }^{6}$ », ce qui peut permettre dans certains cas l'apparition de niches de marchés de spécialité (sous-traitance de spécialité) ou même de conception et de développement. Outre les produits de la transformation des matières plastiques, on y trouve les secteurs de la fabrication de matériel de traitement de l'information, de l'équipement industriel, du travail des métaux et de la fabrication d'instruments et de matériel de précision.

Le développement de l'informatisation et de l'automatisation dans un certain nombre de secteurs a permis de rentabiliser les petites séries (Hardy, 1988). Les TPEI (très petites entreprises industrielles, moins de 10 salariés) se distinguent de leurs homologues non innovantes par la proportion nettement plus grande d'entreprises informatisées ou utilisant l'informatique de production, et par le recours plus important au crédit-bail (Insee Première, nº 268, juillet 1993).

\section{Une offre d'entrepreneurs importante}

La démographie humaine influe sur la démographie des entreprises, une population jeune et qualifiée est plus entreprenante qu'une population vieille et installée. Dans le cas français, l'apparition sur le marché du travail des classes nombreuses de l'après-guerre explique l'accroissement de l'offre d'entrepreneurs potentiels dans la période récente. Cet accroissement est renforcé par :

- le taux de chômage élevé et la difficulté de l'insertion des jeunes diplômés ; leur insertion à un niveau de rémunération médiocre diminue par ailleurs leur coût d'opportunité d'entreprendre et les aides à la création d'entreprises permettent dans un certain nombre de cas de viabiliser un projet;

- une nouvelle mentalité plus favorable à l'entrepreneuriat chez des jeunes mieux préparés par les progrès des formations à la gestion et à la création d'entreprises.

5. Par « niches de marchés », on entend des marchés délaissés par les grandes entreprises, car potentiellement limités au regard des ventes de produits pour des raisons de goûts liées à la culture.

6. Y. Farges, directeur de la recherche et de la technologie chez Pechiney en 1988. 
Un taux de chômage élevé constitue un élément primordial de l'offre d'entrepreneurs, puisque selon les différentes enquêtes réalisées, de $35 \%$ à $50 \%$ des créateurs d'entreprises ont le statut de chômeurs lors de la création de leur entreprise ${ }^{7}$, même si finalement en regard du nombre élevé de chômeurs, la proportion de chômeurs créateurs d'entreprises reste faible (de 1,5\% à $3 \%$ ).

Un engouement pour la création d'entreprise a pu être décelé dans la population. Encensé par les médias, l'entrepreneur a été le héros moderne des années 1980, ainsi que le révèlent les sondages qui enregistrent une croissance du désir de création de sa propre entreprise. Les sondages de l'Institut français d'opinion publique et d'études de marché (IFOP) indiquent une croissance importante du nombre des Français désirant créer leur propre entreprise (3,6 millions en 1983, 5,7 millions en 1987, plus de 10 millions à l'heure actuelle même si moins de $2 \%$ passent à l'acte chaque année). Le développement des formations à la gestion et à la création d'entreprises leur a permis, par ailleurs, de crédibiliser le passage à l'acte. Il y a eu une démocratisation de la connaissance de la gestion qui a longtemps constitué un domaine héréditaire où la transmission du savoir sur le fonctionnement de l'entreprise s'effectuait de père en fils. Parallèlement, les sciences de gestion, après s'être penchées sur les règles de fonctionnement de la grande entreprise, se sont intéressées à la "catégorie hypothétique et improbable de la PME » (Giordanno, 1987) pour se tourner vers l'étude de la création d'entreprises et de la phase de gestation du projet de l'individu-créateur, un certain nombre d'enseignements étant alors consacrés au processus proprement dit de la création d'entreprises (études de marchés, montage juridique et financier, démarrage et montée en puissance de l'activité, etc.)

\section{La nouvelle place des PME}

Toutes les données convergent pour montrer que les PME dans les pays industrialisés ont montré autour des années 1970 un dynamisme du côté de la création d'emplois, de la valeur ajoutée et même des exportations, phénomène qui ne s'est pas démenti depuis. S'agit-il d'un nouvel équilibre, les PME reprenant une partie

7. Il est difficile d'apprécier le nombre exact de chômeurs créant leur entreprise. La dernière enquête de l'INSEE estimait à $27 \%$ le nombre de chômeurs créateurs d'entreprises entre 1990 et 1991. Cependant, dans la réponse à l'enquête, le statut du chômeur se trouvant avec les catégories socioprofessionnelles, un cadre chômeur pouvait très bien s'enregistrer comme cadre ou chômeur; de même, dans les $13,4 \%$ des inactifs qui entreprennent se trouvent probablement un certain nombre de chômeurs découragés (Bonneau et Francoz, 1995). Le dépouillement de l'enquête SINE 1994 sur les créateursrepreneurs d'entreprises du premier semestre 1994 (27 000 individus interrogés sur environ 90000 créateurs-repreneurs dans le premier semestre) montre que $44 \%$ des créateurs-repreneurs étaient des demandeurs d'emplois, $47 \%$ si on ne considère que les créateurs. 
de leur terrain perdu, et comment va évoluer cet équilibre dans un monde où l'on observe et ce de façon paradoxale un renouveau entrepreneurial et une concentration financière croissante?

TABLEAU 1

Nombre d'entreprises répertoriées par la source SIRENE

\begin{tabular}{lcccc}
\hline & Janvier 1982 & Janvier 1987 & Janvier 1992 & Janvier 1996 \\
\hline $\begin{array}{l}\text { Nombre } \\
\text { d'entreprises }\end{array}$ & 2321700 & 2461000 & 2748000 & +3 millions \\
\hline
\end{tabular}

TABLEAU 2

Part des PME manufacturières

(biens intermédiaires [BI], biens d'équipement [BE]

et biens de consommation [BC]) dans le chiffre d'affaires (CA), les exportations et les investissements en France

\begin{tabular}{lccc}
\hline Année & Chiffre d'affaires & Exportations & Investissements \\
\hline 1986 & $39,2 \%$ & $22,7 \%$ & $31,4 \%$ \\
1989 & $41 \%$ & $24,1 \%$ & $36,7 \%$ \\
\hline
\end{tabular}

Source : P. Crosnier, J.P. Francois et T. Lehoucq (1991), Les Chiffres Clés des PMI, Paris, Ministère de l'Industrie et du Commerce extérieur, Services des statistiques industrielles (SESSI).

Entre 1984 et 1988, selon la source du Système unifié de statistiques d'entreprises (SUSE), le nombre de PME (plus de 20 salariés, moins de 500 salariés) augmente de 6030 (passage de 66080 à 72 110). On peut répartir cet accroissement par origine : le solde global 6030 provient de l'excédent des petites entreprises en 1984 (2 400) qui deviennent PME en 1988 sur le mouvement inverse, de l'excédent des grandes entreprises en 1984 (30) qui deviennent PME sur le mouvement inverse et de l'excédent (3 600) du solde des créations et des disparitions d'entreprises (Berthier et Parent, 1994). Au regard du nombre d'emplois, le franchissement du seuil des petites entreprises vers les PME se traduit par un excédent de 33000 emplois, les PME qui se maintiennent comme telles augmentent l'emploi de 107000 ; le solde des grandes entreprises sur ce plan est négligeable, mais celui des créations et disparitions donne une création nette de 165000 postes. Une partie de ces créations-disparitions a cependant pour origine les restructurations, ce qui expliquerait environ la moitié du solde des 165000 emplois.

Selon la source SIRENE, le parc des PME évolue de $25 \%$ entre 1987 et 1992. On constate que le parc des petites et moyennes industries (de 10 à 499 salariés), industries agroalimentaires comprises, crô̂t de 9,13\% puisqu'il passe de 48619 en 1987 à 53070 en 1992. Les petites entreprises industrielles (moins de 20 salariés) occupent par ailleurs une part croissante dans l'industrie. De 1983 à 1991, la part 
de la population occupée dans les PEI a progressé de quatre points, passant de $12 \%$ à $16 \%$ (De Barry et Savoye, 1994).

\section{Les caractéristiques des créateurs d'entreprises}

Qui sont les créateurs d'entreprise? Quelles sont leurs motivations ? Dans quels secteurs créent-ils ? Avec quels moyens ? Voilà quelques questions qui permettent de cerner avec plus de précisions le renouveau entrepreneurial. Déjà, intuitivement, on perçoit que la création d'entreprises ne répondra pas aux mêmes motifs (différence entre la création plus ou moins imposée par la crise, la création voulue, préparée, désirée, assimilée, ou la création résultant de la culture sociale, où l'on créé ou exerce une profession libérale parce que les ascendants exercent une profession indépendante).

\section{Des motivations complexes}

Pour certains économistes, les motivations sont plus complexes que ne le laisse entrevoir la simple occasion de faire un profit de la théorie néoclassique. Outre la remise en cause de la maximisation du profit, certains auteurs pensent que les déterminants de la décision d'entreprendre revêtent des considérations sociales, psychologiques et environnementales. Pour Schumpeter (1939), l'entrepreneur est un être anormal. C'est un «déviationniste» social dont la motivation réside plus dans le goût de la compétition et de l'action que dans des motifs hédonistiques. Baumol (1968), tout en reprenant le rôle de diffusion de l'innovation de l'entrepreneur schumpétérien, pense que les motivations de l'entrepreneur ont beaucoup à voir avec la psychologie sociale et qu'elles s'inscrivent dans un milieu où le développement culturel et la sociologie jouent un rôle. Shapero ${ }^{8}$ a proposé un modèle explicatif de la décision microéconomique d'entreprendre qui retient quatre motivations principales dans l'incitation des individus à créer leur propre entreprise :

- une discontinuité qui exprime une rupture dans la situation de l'individu, celle-ci étant le plus souvent négative (chômage, situation de réfugié, perte d'un parent dans la jeunesse, enfants issus d'un second mariage);

- une disposition à l'action qui se retrouve avec plus ou moins d'intensité chez les individus;

- une disponibilité de ressources financières, humaines et technologiques ;

- une crédibilité de l'acte, qui rend compte à la fois des qualités de l'entrepreneur et de son insertion dans un milieu socioculturel déterminé (insertion dans les réseaux professionnels et entrepreneuriaux locaux).

8. Extension du modèle de Shapero dans Lacasse et Lambert (1988). 


\section{Les résultats des enquêtes}

Les motivations des entrepreneurs apparaissent donc complexes et différentes selon les catégories socioprofessionnelles, les situations au moment de la création et même la localisation de l'individu dans un territoire plus ou moins en difficulté ou qui valorise plus ou moins la fonction entrepreneuriale. Mayer (1977), dans une étude portant sur l'accession des ouvriers à la petite entreprise artisanale et commerciale, montre que ce sont « de jeunes ouvriers que l'âge n'a pas encore conduit à accepter la condition ouvrière, une plus grande intégration au monde ouvrier ou à choisir d'autres moyens de défense (syndicalisme, promotion), qui se mettaient davantage à leur compte ». La recherche d'indépendance concerne plus les populations qualifiées (cadres) $)^{9}$, la maîtrise du destin - qui exprime une position plus défensive est liée au vécu personnel du créateur mais touche plus aussi certaines catégories socioprofessionnelles modestes. La situation du chômage et « gagner de l'argent » n'apparaissent pas en tête des sondages; les réponses peuvent cependant être biaisées dans le but de crédibiliser l'acte d'entreprendre dans le cas de la situation de chômage ou par volonté de dissimulation de ses préférences réelles dans le cas « gagner de l'argent ». Marc (1988) trouve des motivations de l'ordre de la mission sociale chez les entrepreneurs brestois et une volonté de gains plus forte qu'à l'échelle nationale. Il attribue ces motivations aux conditions économiques difficiles du territoire et aux qualifications modestes des créateurs locaux. Dans certains territoires à fort développement endogène, il existe une très forte valorisation de la fonction entrepreneuriale, une culture entrepreneuriale qui explique un taux élevé de créations d'entreprises et un «bon » comportement économique local. La motivation principale des créateurs est l'imitation de la réussite des entrepreneurs en place, un idéal partagé de façon collective sur le territoire.

\section{Des profils divers}

Peut-on définir un entrepreneur type ? Il semble bien que non, puisque l'on trouve des créateurs issus de milieux professionnels différents, de milieux sociaux différents, avec une expérience et une formation différentes, avec des moyens financiers et relationnels différents qui probablement ne vont pas entreprendre dans le même secteur. Peut-on cependant dégager quelques caractéristiques générales qui nous permettent de résumer l'entreprise créée? En fait, il existe une forte reproduction sociale dans la création d'entreprises, qui va déterminer aussi les chances de succès.

Selon une enquête récente déjà citée (Bonneau et Francoz, 1995), les créateurs d'entreprises en France pendant la période 1990-1991 sont plutôt jeunes (66 \% ont

9. Sondages « qui crée en 1985 », « en 1987 » (1988), revue Créez, éditée en collaboration avec l'Agence nationale de la création d'entreprise (ANCE). 
moins de 40 ans), plutôt diplômés (47\% ont une formation équivalente ou supérieure au baccalauréat contre 22 \% dans la population française totale), les chômeurs, les employés et les ouvriers représentent plus de la moitié des créateurs. Bien entendu, le profil du créateur, en particulier sa formation initiale, va déterminer le secteur de la production; les formations telles que le brevet d'enseignement professionnalisé (BEP) ou le brevet d'étude de premier cycle (BEPC) orientent vers les métiers de production de biens (industrie et construction), les professions supérieures vers le tertiaire (en délaissant toutefois le commerce).

La plupart des créateurs choisissent une activité connue, que ce soit par la pratique ou la délivrance d'un diplôme correspondant à un métier précis. Hormis les caractéristiques sectorielles, la formation générale du créateur favorise la pérennité de l'entreprise, de même qu'une expérience dans l'entreprise, en particulier dans les fonctions d'encadrement qui ont initié le créateur à la prise de décision et, dans un certain nombre de cas, à la gestion de l'entreprise. La jeunesse dans l'échantillon étudié apparaît alors comme un facteur négatif, en aggravant le risque de faillite, les jeunes créant pour échapper au chômage et n'ayant peu ou pas d'expérience professionnelle.

\section{Une forte reproduction sociale}

Kombou (1986) a montré que la catégorie sociale dont est issu le créateur détermine en partie le secteur de la création et la taille de l'entreprise créée, de même que l'intensité du passage à l'acte d'entreprendre, puisque plus d'un créateur sur deux dans son échantillon est issu d'un milieu entrepreneurial. Ce résultat est confirmé par un grand nombre d'autres études ${ }^{10}$. La création issue d'un milieu entrepreneurial se fait dans de plus grandes classes de taille. Par le montant des capitaux propres, par le soutien de la famille, par l'imprégnation d'une culture entrepreneuriale, elle augmente les probabilités de survie de la jeune entreprise. Cette création issue d'un milieu entrepreneurial est importante, puisque environ $50 \%$ des créateurs d'entreprises sont issus ou appartiennent au milieu des «indépendants », professions libérales et petits patrons. La réussite dans l'entreprise est aussi une incitation à créer d'autres structures juridiques permettant soit de diversifier l'activité, soit d'installer les descendants ; les chances de survie de la nouvelle entreprise sont alors bien meilleures.

En fin de compte, on peut distinguer deux populations typiques de créateurs d'entreprises :

- les créateurs formés, de condition sociale élevée, bien souvent issus d'un milieu entrepreneurial ayant une perception large du monde économique

10. Shapero, dans Keeble et Wewer (1986), Kombou et Kochansky (1988), Gollec et Lauhlé (1987), Viennet (1988), Ghazali, Ghosh et Tay (1994). 
qui les entoure, et dont la création repose sur des occasions d'affaires et sur des choix clairement raisonnés (motivations principales : l'indépendance et la perception d'un marché).

- les créateurs de conditions sociales moins élevées qui entreprennent, soit par contrainte, soit par désir de maîtrise du destin, soit par volonté d'accéder à un statut social plus élevé.

\section{La mesure du renouvellement}

Conceptuellement, l'évolution sectorielle du stock d'entreprises est égale au solde du nombre des créations et des disparitions d'entreprises pendant une période donnée pour le secteur considéré. Les sources statistiques sur les stocks et les flux d'entreprises présentent un certain nombre de limites ${ }^{11}$ liées à la qualité des sources statistiques et à la signification des flux d'entreprises.

Devant ces limites, une comparaison de l'évolution des stocks d'établissements et du nombre des effectifs salariés par secteurs nous permet de créer une typologie sectorielle, certes imparfaite mais, après vérification par des études monographiques sectorielles, non dénuée de pertinence.

\section{Typologie sectorielle du renouvellement des établissements et des entreprises}

La réponse du tissu productif à une augmentation de la demande peut prendre plusieurs formes à une échelle sectorielle. On peut en effet supposer :

- une réponse des entreprises existantes du secteur considéré à cette augmentation de la demande, soit par la création de nouveaux établissements, soit par l'augmentation de la production dans les établissements déjà existants ;

- une réponse de la part d'entreprises d'autres secteurs, en général proches, qui vont en profiter pour se diversifier en créant des établissements dans le secteur. Schwalbach (1987) montre que, dans l'industrie allemande, les entrées par diversification s'expliquent plus par le souci de réaliser des économies d'envergure ${ }^{12}$ que par le désir de réduire le risque de son activité;

- une réponse par les importations;

- une réponse par la création ex nihilo d'entreprises ; les nouvelles entreprises industrielles présentent alors un potentiel de développement et de

11. Annexe II.

12. Les économies d'envergure sont les économies liées à la politique de diversification de l'activité de la firme. 
créations d'emplois nettement supérieur à celui des autres secteurs (Bonneau, 1996).

L'allocation optimale des ressources entre les différents secteurs s'effectue par l'apparition de nouveaux établissements et de nouvelles entreprises dans les secteurs à taux de profits élevés. Mais le taux de profit peut aussi rendre compte d'une concurrence imparfaite sur les marchés et de la présence de barrières à l'entrée susceptibles de décourager les entrants. Il semble donc judicieux d'étudier la régulation sectorielle du nombre des établissements et des entreprises en séparant les secteurs concentrés des secteurs concurrentiels, puisque les déterminants ne seront pas identiques ou tout au moins n'agiront pas avec la même intensité ${ }^{13}$. Il existe alors une régulation du nombre des entreprises propre à chaque secteur. Les taux de renouvellement d'entreprises sont très différents selon les secteurs, en raison principalement de degrés de concentration distincts, de taux de sous-traitance différenciés et d'une variabilité de la demande plus ou moins forte. Les défaillances seront aussi plus ou moins importantes selon les secteurs, les secteurs à forts taux de création se caractérisant en général par de forts taux de défaillance et, donc, par un indice de renouvellement élevé.

Il est possible, à l'aide d'indicateurs économiques, de distinguer quatre cas de régulation du nombre des établissements et des entreprises par secteur ${ }^{14}$.

Le premier cas concerne les secteurs concurrentiels en perte de vitesse, pour lesquels on observe une augmentation du nombre des faillites d'entreprises marginales, une diminution des stocks d'établissements et d'entreprises avec, cependant, la possibilité d'un fort renouvellement si les barrières à l'entrée (mesurées par la taille moyenne en effectifs salariés) sont faibles.

Le deuxième cas vise les secteurs concurrentiels en croissance, qui verront leurs stocks d'établissements et d'entreprises augmenter, particulièrement dans les

13. La concentration n'est pas toujours gage de concurrence imparfaite selon la théorie des marchés contestables. Dans les faits, cependant, il semble que cette théorie s'applique à quelques secteurs bien particuliers.

14. La classification des secteurs dans les quatre cas possibles prend en compte:

- la croissance du secteur appréciée par l'évolution des effectifs salariés de l'UNEDIC pendant la période 1977-1991.

- les barrières à l'entrée estimées par la taille moyenne en effectifs salariés des établissements de l'UNEDIC et son évolution par secteur pendant la période 1977-1991.

- la structure du marché, évaluée par le niveau de concentration sectorielle (état de la concentration en 1985 pour les secteurs industriels, source SESSI, part des quatre premières et part des 10 premières dans les ventes).

- l'intensité du renouvellement apprécié par les taux de création et de défaillances d'entreprises ; source des flux (CEPME); source des stocks (établissement UNEDIC), pour la période 1979-1985. Les taux présentent une grande constance. 
secteurs à faibles barrières à l'entrée, les disparitions d'entreprises (cessations volontaires de l'activité et défaillances) étant en croissance du fait du nombre élevé de créations d'entreprises.

Le troisième cas prend en compte les secteurs concentrés qui mettent en place des barrières stratégiques à l'entrée (politiques de prix, de capacités excédentaires, commerciales) qui constituent un frein à la création d'entreprises. Il existe alors un faible renouvellement du tissu productif.

Enfin, le dernier cas renvoie à certains secteurs concentrés qui ont une régulation des flux d'entreprises plus subtile combinant, d'une part, les occasions d'entrée dans les niches de marché ou les niches technologiques ${ }^{15}$ et, d'autre part, les restructurations.

\section{Un renouvellement différent selon les secteurs}

Dans le premier cas, on observe une réduction des effectifs salariés et, en général, une réduction du nombre des établissements et des entreprises. Parmi les secteurs à faibles barrières à l'entrée, il est possible de classer les secteurs des articles d'habillement, des articles de cuir, des machines-outils, de la fabrication d'appareils ménagers, des produits du travail mécanique du bois (avec une faible croissance du nombre des établissements et des taux de création très élevés) et des industries diverses (avec, là aussi, une légère croissance du nombre des établissements et des taux de création forts mais légèrement plus faibles que pour le secteur des produits du travail mécanique du bois).

Dans les secteurs à taille moyenne en effectifs salariés relativement élevée, on trouve les secteurs des produits de la première transformation de l'acier (avec, cependant, une légère croissance du nombre des établissements), les produits de la fonderie, de la fabrication de machines agricoles et des industries textiles.

Le deuxième cas représente les secteurs industriels à forte croissance et à évolution favorable de la demande. On trouve, pour les secteurs à faibles barrières à l'entrée, le secteur de l'imprimerie, presse et édition avec une croissance forte du nombre des établissements mais une faible croissance du nombre des effectifs salariés : la taille moyenne en effectifs salariés diminue fortement et le taux de création est très élevé. Les produits de la transformation des matières plastiques,

15. Les niches technologiques se définissent par l'existence de marchés délaissés par les grandes entreprises en raison de l'utilisation de technologies très pointues reposant sur la connaissance de quelques spécialistes, généralement issus de centres de recherche privés ou publics et qui ont décidé d'exploiter à leur compte certains domaines de leurs recherches. Bien souvent, la grande entreprise aide ces jeunes entreprises, ce qui lui permet de suivre l'avancement de la mise en œuvre des technologies (Nijkamp et Giaoutzi, 1988). 
les machines d'équipement industriel et les produits du travail des métaux ont une régulation du tissu productif similaire au secteur précédent avec, cependant, des taux de création plus faibles. Pour ces secteurs, il existe des niches de marché. Le secteur pain et pâtisserie est remarquable par sa très faible taille moyenne en effectifs salariés, qui augmente néanmoins pendant la période. Cela retrace la mise en place de grosses unités de production de fabrication industrielle de pains, de brioches, etc. (la croissance du nombre des établissements est faible et les taux de création sont faibles par rapport à la masse des boulangers).

Parmi les secteurs à fortes barrières à l'entrée, on peut recenser le secteur de la parachimie qui présente une très faible croissance du nombre de ses établissements et des taux de création relativement faibles. Le secteur des produits pharmaceutiques se concentre avec l'augmentation de sa taille moyenne en effectifs salariés et la diminution de son nombre d'établissements, les entreprises existantes s'appropriant la plus grande partie de la croissance et les taux de création étant très faibles.

Le troisième cas représente les secteurs où peuvent jouer les stratégies des grandes entreprises. On y trouve aussi des conditions de la demande plus ou moins favorables. De fait, dans un certain nombre de ces secteurs concentrés, les conditions de la demande ont plutôt évolué défavorablement et il n'y a pas eu lieu de mettre en place des stratégies de barrières à l'entrée. On peut citer les secteurs concentrés du minerai de fer (forte décroissance des effectifs et des établissements, avec la fermeture des mines), de la sidérurgie, de l'extraction et de la préparation de minerais non ferreux, de la production de minéraux divers, de la construction navale et de l'industrie du caoutchouc.

On peut classer dans le quatrième cas les secteurs concentrés qui ont connu une forte croissance en effectifs salariés et en nombre d'établissements avec l'existence de niches technologiques ou de niches de marché favorables à la création d'entreprises. C'est le cas du secteur de la fabrication de machines de bureau et de matériel de traitement de l'information et, dans une certaine mesure, du secteur de la construction aéronautique (avec, dans ces deux cas, probablement une politique active «d'essaimage technologique»).

\section{La diversité du renouvellement des établissements et des entreprises dans l'espace}

Diverses combinaisons d'urbanisation, de concentration des activités économiques, de renouvellement des activités motrices, de culture industrielle, d'histoire, de migrations avec leurs effets d'entraînement et d'aides économiques, rendent compte du développement différencié dans l'espace. Pendant une période déterminée, ce développement différencié peut être expliqué par : 
- l'urbanisation et les économies d'agglomération (économies externes) qui représentent les avantages que les entreprises tirent de leur regroupement dans l'espace ;

- la composition des activités sur le territoire, lequel est plus ou moins spécialisé dans des secteurs en déclin ou en croissance ;

- le renouvellement des activités motrices et la modification des facteurs de localisation de ces activités (Bonnet, 1995);

- le «potentiel local» qui mesure à travers certaines variables socioéconomiques le dynamisme propre au territoire (intensité entrepreneuriale, variables démographiques telles que l'accroissement naturel et le solde migratoire de la population, capacité à mobiliser l'épargne dans des projets locaux, goût du travail, comportement sociétal, etc.).

Les migrations et leurs flux de revenus associés peuvent aussi contribuer à différencier le développement dans l'espace, il en va de même de l'action volontariste des pouvoirs publics dans leur politique d'aménagement du territoire et de développement régional.

La crise de 1973 constitue une rupture dans la concentration croissante de la production dans quelques unités de production et dans le développement des régions de vieille industrialisation. On constate, en effet, un renouvellement des secteurs en croissance qui s'accompagne d'un renouvellement marqué des entreprises, en particulier par la création d'entreprises nouvelles, celles-ci ne se distribuant pas avec la même intensité sur tout le territoire, certaines régions enregistrant des taux de création d'entreprises plus importants ${ }^{16}$. Les études montrent que si le dynamisme dans la création d'entreprises varie fortement selon les secteurs, il existe une spécificité régionale indéniable ${ }^{17}$.

\section{Les résultats d'une analyse structurelle et résiduelle}

En suivant Ashcroft, Love et Malloy (1991), il est possible de calculer des indices de performance relative, comme le rapport du nombre d'employeurs de la région considérée à la date finale sur le nombre d'employeurs que l'on obtiendrait si la

16. Ces régions connaissent aussi des taux de défaillance plus élevés, mais le solde est positif. Ces taux de création plus importants proviennent de la modification des facteurs de localisation des activités en croissance et des soldes migratoires qui ont privilégié en particulier les régions du Sud, mais aussi de l'existence dans certains territoires d'une régulation locale favorable à l'entrepreneuriat.

17. «Le dynamisme des régions vu à travers la création d'entreprises », note pour messieurs les directeurs régionaux à l'attention des chefs de services d'études, INSEE, direction générale, octobre 1992. 
région avait connu la même évolution sectorielle du nombre des employeurs que la France pendant la période considérée.

\section{TABLEAU 3}

Indice de performance relative de l'évolution du nombre d'employeurs de l'UNEDIC

\begin{tabular}{|c|c|c|c|c|}
\hline & \multicolumn{2}{|c|}{ Industrie } & \multicolumn{2}{|c|}{ Tertiaire } \\
\hline & Indice & Classement & Indice & Classement \\
\hline Corse & 1,425 & 1 & 1,497 & 1 \\
\hline Languedoc-Roussillon & 1,162 & 2 & 1,129 & 7 \\
\hline Pays-de-la-Loire & 1,155 & 3 & 0,916 & 19 \\
\hline Poitou-Charentes & 1,138 & 4 & 0,957 & 14 \\
\hline Alsace & 1,136 & 5 & 1,336 & 2 \\
\hline Bretagne & 1,132 & 6 & 1,221 & 3 \\
\hline Midi-Pyrénées & 1,131 & 7 & 1,136 & 6 \\
\hline Aquitaine & 1,129 & 8 & 1,140 & 5 \\
\hline Bourgogne & 1,109 & 9 & 1,020 & 8 \\
\hline Basse-Normandie & 1,082 & 10 & 0,987 & 10 \\
\hline Provence-Alpes-Côte-d'Azur & 1,057 & 11 & 0,899 & 22 \\
\hline Centre & 1,056 & 12 & 1,014 & 9 \\
\hline Rhônes-Alpes & 1,043 & 13 & 1,145 & 4 \\
\hline Lorraine & 1,042 & 14 & 0,903 & 21 \\
\hline Champagne-Ardennes & 1,022 & 15 & 0,941 & 15 \\
\hline Haute-Normandie & 1,017 & 16 & 0,976 & 11 \\
\hline Franche-Comté & 0,973 & 17 & 0,965 & 13 \\
\hline Nord-Pas-de-Calais & 0,957 & 18 & 0,923 & 17 \\
\hline Picardie & 0,933 & 19 & 0,906 & 20 \\
\hline Auvergne & 0,930 & 20 & 0,930 & 16 \\
\hline Limousin & 0,881 & 21 & 0,966 & 12 \\
\hline Île-de-France & 0,821 & 22 & 0,917 & 18 \\
\hline
\end{tabular}

On peut ainsi différencier les types de développement que l'on peut mettre en évidence dans le cas français, avec pour les régions du Sud, un développement plutôt axé sur l'attractivité forte de ces régions par rapport à la population et aux activités ; pour les anciennes régions industrialisées, un développement bridé sinon contrarié par leurs spécialisations sur des activités en perte de vitesse; pour les régions de l'Ouest, un développement endogène favorisé par une forte culture entrepreneuriale; pour l'île-de-France, métropole aux fonctions supérieures diverses qui concentre les services les plus qualifiés, un développement peut-être 
plus qualitatif que quantitatif, qu'il faudrait plutôt apprécier au regard de la valeur ajoutée brute qu'à celui des effectifs ${ }^{18}$.

\section{Une interprétation de l'entrepreneuriat à l'échelle spatiale}

W. Isard (1956) avait mis en évidence «les gains de productivité attribuables à l'agglomération géographique des populations ou des activités économiques » par un processus circulaire de localisation des activités productives. Les agglomérations, en assurant un vaste marché local, attirent de nouvelles industries qui élargissent encore le marché local, etc. De nombreux travaux ${ }^{19}$ montrent que la création d'entreprise est favorisée par l'urbanisation en particulier par un effet de la demande qui se manifesterait dans les grandes agglomérations surtout dans les secteurs des services aux ménages et aux entreprises et moins dans les secteurs du commerce et de l'artisanat. On trouve aussi un effet de l'urbanisation dans les faillites plus élevées suscitées par les créations nombreuses et dans des durées de vie moyenne probablement moins élevées. L'île-de-France présente alors des taux élevés de création rapportés aux firmes existantes et à la population salariée ${ }^{20}$.

Par ailleurs, les études anglaises mettent en évidence une liaison entre les propriétaires de maisons individuelles et l'intensité entrepreneuriale, les auteurs attribuant généralement cette relation au fait que la propriété sert de caution dans le financement de la nouvelle entreprise et que la propriété est un indicateur de la richesse locale qui peut influencer la demande locale.

Dans le cas français, Bernard Guesnier trouve une relation négative entre les propriétaires de maisons individuelles et l'intensité entrepreneuriale, mais par contre une liaison positive avec le nombre de résidences secondaires. Selon l'auteur, les relations doivent être analysées et explicitées à partir d'une segmentation plus fine des espaces retenus (dans l'étude, les départements français). L'auteur retient alors :

18. Pour la Corse, la population très faible des employeurs et l'influence des subventions publiques soutenant l'économie locale surreprésentent l'intensité entrepreneuriale et doivent inciter à la plus grande prudence quant à l'interprétation des résultats. Inversement pour l'île-de-France, la présence d'un nombre important de sièges sociaux sousestime son poids économique réel. On constate en effet une désindustrialisation relative de l'île-de-France, qui selon la source UNEDIC ne représente plus que $20,47 \%$ des effectifs salariés français de l'industrie en 1991 contre $22,47 \%$ en 1977 ; cette relative désindustrialisation n'est pas contrebalancée par l'évolution du tertiaire, puisque l'Îlede-France représentait 32,04\% en 1977 des effectifs salariés de ce secteur contre 30,06\% en 1991. Seul le secteur du bâtiment et des travaux publics (BTP) connaît un accroissement de la part relative de l'île-de-France puisqu'il passe de $22,31 \%$, preuve s'il en est du dynamisme aménageur de cette région.

19. Ashcroft, Love et Malloy (1991), Keeble et Walker (1993), Guesnier (1994).

20. Période 1986-1991, Sources : SIRENE, ANCE dans B. Guesnier (1994), «Régional variations in new firm formation in France », Régional Studies, vol. 28. 
- les grandes zones urbaines,

- les zones résidentielles attractives,

- les zones rurales industrialisées,

- les milieux technologiques,

- les zones localement intégrées.

Olivier Bouba-Olga et Bernard Guesnier ${ }^{21}$ ont effectué récemment des calculs selon la méthode structurelle et résiduelle à l'échelle des zones d'emplois. En classifiant le développement des différentes zones, ils ont pu mettre en évidence les formes du dynamisme spatial, en distinguant différentes configurations spatiales (noyau - une seule zone d'emplois (en général urbaine) dynamique -, noyau avec halos - le dynamisme de la zone d'emplois principale diffuse dans les zones d'emplois contiguës -, halos diffus - pas de zone d'emplois dominante mais un ensemble de zones d'emplois contiguës à «bon » comportement -, etc.). Ils ont par ailleurs testé le dynamisme des zones d'emplois selon les différentes hypothèses théoriques ; de type MAR (Marshall, Arrow, Romer), avec une spécialisation forte qui génère un stock de connaissances communes et des externalités, ou selon la théorie évolutionniste, où, au contraire, des spécialisations diverses permettent aux entreprises de garder «une flexibilité comportementale dynamique ». Les résultats révèlent que les zones de multiples spécialisations ont présenté des résultats plus positifs que les zones à spécialisation excessive dans un seul domaine d'activité.

En outre, les études montrent une corrélation entre la présence de petites entreprises et l'intensité entrepreneuriale. Cette relation s'interprète de différentes façons; on trouve :

1. la perte de vitesse des régions de vieille industrialisation spécialisées dans des secteurs en déclin et à taille moyenne en effectifs salariés élevée ;

2. une période favorable à l'entrepreneuriat, où l'apprentissage de la fonction entrepreneuriale est facilitée dans de petites organisations ;

3. l'existence de districts industriels ${ }^{22}$.

21. «Dynamiques des systèmes localisés de production et cadre spatial d'analyse », allocution présentée au colloque «Dynamiques industrielles, dynamiques territoriales », Toulouse, 30-31 août et $1^{\text {er }}$ septembre 1995.

22. Historiquement, les districts industriels marshalliens se caractérisent par la concentration efficace de petites entreprises spécialisées dans différentes fonctions du processus de production d'une industrie particulière. L'existence d'une population ouvrière qualifiée crée alors les conditions d'un apprentissage local du métier et de l'innovation dans l'ensemble de la population et en particulier chez les enfants « qui respirent l'atmosphère industrielle ». A. Marshall (1961), Principles of Economics, $1^{\text {re }}$ édition 1890, $9^{\mathrm{e}}$ édition, Londres, McMillan. 
Enfin, cette relation rend compte de la forte attractivité de certains territoires, puisque les migrations vers les régions du Sud s'accompagnent de la création de son propre emploi dans certains cas et, en tout état de cause, sont sources de revenus, donc, de développement induit (personnes âgées dans la région Provence-AlpesCôte-d'Azur, ou même chômeurs préférant chômer au soleil de Toulouse ou de Montpellier).

La relation entre le taux de chômage et l'intensité entrepreneuriale à l'échelle régionale n'est donc pas si simple. On trouve des régions de vieille industrialisation en reconversion à faible taux de création d'entreprises nouvelles et à fort taux de chômage, des régions attractives en développement à fort taux de création d'entreprises nouvelles mais aussi à fort taux de chômage, et des régions ou des territoires de «culture entrepreneuriale» qui présentent un faible taux de chômage et des créations importantes.

\section{Une faible pérennité des nouvelles entreprises}

Les pouvoirs publics encouragent, de façon générale, les aides à la création d'entreprises comme solution de rechange au chômage, en particulier par les aides aux chômeurs créateurs ou repreneurs d'entreprises. Le nombre élevé d'entreprises nouvelles entraîne, cependant, de nombreuses cessations d'activité au cours des premières années de vie de l'entreprise. Parmi ces cessations d'activité, un certain nombre d'entreprises déposent leur bilan. Une politique de maintien des entreprises en place doit alors s'interpréter comme une politique substituable à la création d'entreprises. Il peut donc exister un conflit à vouloir favoriser la création d'entreprises et en même temps préserver à tout prix les entreprises de la faillite. Le législateur a, en fait, par ses lois sur la préservation du tissu productif (lois sur la défaillance de 1984-1985) rendu paradoxalement plus difficile la création de nouvelles entreprises, les banques étant réticentes à prêter. Une modification de la Loi sur les défaillances est intervenue en 1994, redonnant aux créanciers certaines garanties.

\section{Les incitations à la création d'entreprises}

Deux facteurs principaux peuvent expliquer la variation d'intensité dans la création d'entreprises des chômeurs créateurs d'entreprises :

- la diminution des allocations de chômage : celles-ci ont vu leur montant décroître et leurs conditions d'attribution devenir plus sévères. De $100 \%$ du salaire brut antérieur au début de la crise (ce qui pouvait représenter $110 \%$ du salaire net dans certains cas de stage de formation), l'allocation va décroître à $65 \%$ puis à $57 \%$ du salaire brut antérieur avec l'apparition d'un délai de carence, d'un temps minimal de travail dans les six mois précédents et d'une forte dégressivité dans le temps (Grangeas et Lepage, 1993); 
- l'évolution des dispositifs d'aides à la création d'entreprises pour les chômeurs créateurs ou repreneurs d'entreprises ${ }^{23}$.

\section{TABLEAU 4}

Nombre et proportion de chômeurs aidés dans le total des créations et reprises d'entreprises

\begin{tabular}{l|c|c|c|c|c|c|c|c|c|c|c|c|c|c|c}
\hline & 1981 & 1982 & 1983 & 1984 & 1985 & 1986 & 1987 & 1988 & 1989 & 1990 & 1991 & 1992 & 1993 & 1994 & 1995 \\
\hline $\begin{array}{l}\text { Nombre de } \\
\text { chômeurs } \\
\text { créateurs ou } \\
\begin{array}{l}\text { repreneurs } \\
\text { d'entreprises } \\
\text { ayant } \\
\text { bénéficié } \\
\text { de l'aide }\end{array}\end{array}$ & 24700 & 33900 & 31400 & 29000 & 57600 & 72000 & 63000 & 56000 & 52700 & 44000 & 44000 & 50000 & 53500 & 80000 & 88300 \\
$\begin{array}{l}\text { Pourcentage } \\
\text { du nombre } \\
\text { total des } \\
\text { créations } \\
\text { et reprises (\%) }\end{array}$ & 10,2 & 14,8 & 15,0 & 13,3 & 23,6 & 27,1 & 23,0 & 20,1 & 20,1 & 17,1 & 19,2 & 22,5 & 24,4 & 34,3 & 39,1 \\
\hline
\end{tabular}

Si l'on comptabilise en nombre d'entreprises aidées, on trouve un pourcentage d'entreprises aidées par rapport au total des créations et reprises légèrement moins important (de $1 \%$ à $2 \%$ selon les années) puisqu' un certain nombre d'entreprises aidées regroupent plusieurs bénéficiaires. Le nombre élevé des créateurs repreneurs d'entreprises aidés en 1995 recoupe deux périodes contrastées ; une forte croissance jusqu'en août 1995 (48 \% de hausse dans le premier semestre par rapport au semestre de l'année précédente) suivi d'une baisse de $16 \%$ au cours du second semestre, conséquemment aux conditions plus restrictives de la législation.

En fait, on constate que si les dispositifs plus ou moins favorables à la création d'entreprises par les chômeurs accroissent le nombre d'entreprises aidées, ils ont probablement un effet moindre sur le nombre de chômeurs créant leur entreprise, qui, dans un grand nombre de cas, de toute manière seraient passés à l'acte. Les pouvoirs publics encouragent de façon plus générale les aides à la création d'entreprises comme solution de rechange au chômage.

\section{Une forte cessation d'activité des jeunes entreprises dans les premières années de vie}

De $50 \%$ à $55 \%$ des cessations d'entreprises concernent des entreprises de moins de cinq ans (Francoz, 1996). Par ailleurs, en 1993, 55 \% des entreprises défaillantes ont moins de cinq ans d'ancienneté alors que le poids de cette classe d'âge dans

23. Annexe III. 
l'ensemble du parc n'est que de $38 \%$. Ainsi, les jeunes entreprises sont plus nombreuses à être défaillantes. Le faible coût d'entreprendre (au sens large, coût financier mais aussi coût de formation, de qualification) dans certains secteurs est un élément important du passage à l' acte d'entreprendre. C'est le cas, par exemple, des secteurs des hôtels-cafés-restaurants et du commerce, qui présentent aussi des particularités de renouvellement important (changement de localisation des propriétaires avec ventes et rachats de fonds de commerce).

TABLEAU 5

Le devenir des entreprises créées ou reprises

\begin{tabular}{lccccccc}
\hline $\mathbf{1 9 8 7 - 1 9 9 2}$ & $\begin{array}{c}\text { Industries } \\
\text { agro- } \\
\text { ali- } \\
\text { mentaires }\end{array}$ & $\begin{array}{c}\text { Hôtels- } \\
\text { cafés- } \\
\text { restau- } \\
\text { rants }\end{array}$ & $\begin{array}{c}\text { Services } \\
\text { aux } \\
\text { ménages }\end{array}$ & $\begin{array}{c}\text { Industrie } \\
\text { Service } \\
\text { aux } \\
\text { entre- } \\
\text { prises }\end{array}$ & $\begin{array}{c}\text { Transports Commerce } \\
\text { et télé- } \\
\text { commu- } \\
\text { nications }\end{array}$ & $\begin{array}{c}\text { Bâtiment } \\
\text { et } \\
\text { travaux } \\
\text { publics }\end{array}$ \\
\hline $\begin{array}{l}\text { Taux de } \\
\text { survie au }\end{array}$ \\
$\begin{array}{l}5 \mathrm{c} \text { anniversaire } \\
62 \%\end{array}$ & $44 \%$ & $66 \%$ & $53 \%$ & $54 \%$ & $53 \%$ & $44 \%$ & $49 \%$ \\
\hline
\end{tabular}

Source : D. Francoz et J. Bonneau (1994), «Le devenir des entreprises créées en 1987 », Insee Première, avril, 4 pages.

Seulement environ $50 \%$ des entreprises nouvelles sont encore en vie cinq ans plus tard. Ce taux de survie se différencie fortement par secteur tout en étant relativement stable dans le temps.

En introduisant un taux de continuité économique (c'est-à-dire qu'un certain nombre de cessations sont suivies de reprises), la hiérarchie des taux de survie entre les secteurs est moins forte; les services aux ménages sont alors devancés par les secteurs des industries agroalimentaires et des hôtels-cafés-restaurants. On remarquera que, selon une étude récente, ces taux de survie varient peu dans les pays industrialisés en Europe ${ }^{24}$, où environ $65 \%$ des nouvelles entreprises passent le cap des trois ans et $50 \%$, le cap des cinq ans. Par la suite, les taux de défaillance tendent à être identiques à ceux des entreprises plus âgées.

\section{Les facteurs spécifiques de la pérennité des jeunes entreprises}

On observe une forte variation des taux de croissance chez les jeunes entreprises, variation qui retrace en fait la zone de «haute mortalité infantile», zone de fort risque de défaillance où l'entreprise et surtout le créateur doivent confronter le projet à la réalité. Hormis le secteur de la création et le créateur, un certain nombre d'autres facteurs vont cependant différencier la pérennité des entreprises :

24. «Entreprise Demography », dans Entreprises in Europe, Third Report, Publications Eurostat, octobre 1994. 
- les reprises d'entreprises apparaissent plus solides ;

- le taux de survie augmente avec la taille de l'entreprise, mais avec un effet de seuil, à partir de 10 salariés et jusqu'à 19 salariés, niveau auquel les taux de survie diminuent; les entreprises de cette taille s'attaquent en effet à des marchés plus difficiles ;

- le montant des capitaux initiaux propres est important, car il permet à l'entreprise de passer certains caps difficiles. Les projets de plus de $500000 \mathrm{FF}$ de capitaux initiaux sont beaucoup plus robustes que les projets requérant un investissement initial inférieur; $77 \%$ d'entre eux sont encore en exploitation à quatre ans révolus contre $60 \%$ environ pour les projets de plus faible taille (Viennet, 1990); malheureusement, seulement $5 \%$ des créateurs parviennent à rassembler une telle somme ;

- les relations avec les autres entreprises, qu'il s'agisse d'un donneur d'ordres, d'un fournisseur, d'une clientèle déjà démarchée, d'entreprises prêtes à fournir à la nouvelle entreprise un soutien logistique, améliorent les chances de survie;

- le recours aux conseils (Points Chances) améliore sensiblement les chances de survie de l'entreprise qui s'en trouvent doublées.

Par ailleurs, les entreprises sociétaires ont pris une part croissante dans le nombre de créations de nouvelles entreprises. Elles apparaissent plus fragiles par rapport à la défaillance, car la dissociation entre le patrimoine de l'entrepreneur et celui de l'entreprise favorise le dépôt de bilan. Cependant, les entreprises individuelles enregistrent une plus grande proportion de cessations volontaires de l'activité et, dans le cas de reprise de l'activité, on constate une transformation assez courante de l'entreprise individuelle sous la forme juridique sociétaire ${ }^{25}$. Dans la période récente, le stock de sociétés a donc progressé pendant que celui des entreprises individuelles diminuait ${ }^{26}$.

Plus que la création, c'est la survie des jeunes entreprises qui est importante. Les pouvoirs publics, sans négliger l'importance des créations d'entreprises, se sont effectivement inquiétés de la croissance très forte du nombre des défaillances d'entreprises pendant la période de forte récession en 1993. Toute politique favorable à la

25. Depuis la loi Madelin, on a cependant enregistré une croissance de la proportion d'entreprises individuelles dans le total des entreprises créées ou reprises avec l'aide aux chômeurs créateurs ou repreneurs d'entreprises (ACCRE).

26. En 1994, $71 \%$ des entreprises ont le statut d'entreprise individuelle. Installées pour l'essentiel en province, elles font travailler plus de trois millions de personnes et réalisent environ $15 \%$ de la valeur ajoutée en France. La plupart n'ont pas de salariés mais les 50000 qui en emploient en comptent trois en moyenne. 
création d'entreprise peut buter sur la demande dans le secteur considéré ou même sur l'offre d'entrepreneurs. On peut supposer qu'il n'y a pas une offre illimitée d'entrepreneurs capables de coordonner les activités de manière efficiente.

\section{Le maintien des entreprises en place peut-il remplacer la création d'entreprises?}

Les orientations du droit des faillites et les mesures d'aides prises en faveur des entreprises en difficulté oscillent entre la nécessité d'une sanction économique des firmes non rentables et la prise en compte des coûts sociaux et économiques que peuvent entraîner les faillites. En période de crise, les pouvoirs publics accordent une importance accrue au maintien du tissu productif, ce qui n'est pas sans contradiction avec les aides à la création d'entreprises. Il apparaît en effet que les défaillances s'expliquent principalement par des fautes de gestion et de management. Les causes macroéconomiques des défaillances d'entreprises (politique du crédit, activité économique, progrès technique, mortalité précoce) éliminent en premier lieu les entreprises mal gérées. Cependant, de même qu'il faut aider les nouvelles entreprises à passer la zone de haute mortalité, ne faut-il pas préserver en partie le tissu productif ? La réponse est ambiguë. La croissance des créations d'entreprises signifie certes un renouvellement du tissu productif mais elle rend compte par ailleurs de la crise et du chômage. Dans ces conditions, l'aide aux entreprises en difficulté ne peut-elle pas se concevoir comme un substitut à la création d'entreprises par les chômeurs?

La réforme de la loi portant sur les procédures collectives en date du 10 juin 1994 s'articule autour de trois principaux axes :

- le développement de la prévention par le renforcement du règlement à l'amiable ;

- l'accélération et la simplification de la procédure judiciaire;

- le renforcement des droits des créanciers.

Le premier axe se justifie par l'idée selon laquelle il est plus efficace de sauver les entreprises en traitant de leurs difficultés par la voie préventive, étant donné l'inefficacité des procédures de redressement judiciaire (moins de $10 \%$ des entreprises ayant bénéficié de plans de redressement étaient effectivement redressées avec la précédente législation). Le premier objectif de cette réforme est donc de renforcer la prévention des difficultés des entreprises en vue d'un meilleur traitement ${ }^{27}$. La

27. Il s'agit d'éviter les phénomènes de faillites en cascade. L'objectif de la loi de 1994 est clairement la réduction des effets de contagion (mesures pour la protection des fournisseurs). 
procédure a également été simplifiée et accélérée, puisque le tribunal a la faculté de prononcer directement la liquidation judiciaire pour éviter l'ouverture de périodes d'observations inutiles. Enfin, les droits des créanciers ont été renforcés, dans le but de consolider la coopération entre l'entreprise et ses créanciers ${ }^{28}$.

En 1994, 260000 salariés ont été concernés par une défaillance, auxquels il faudrait ajouter plus de 30000 non-salariés d'entreprises n'employant aucun salarié ou n'en ayant qu'un seul. Le nombre de chômeurs en résultant est cependant moins élevé, car il faut prendre en compte que les $10 \%$ des entreprises qui continuent leur activité sont de taille élevée (on constate néanmoins une augmentation dans la période récente de la taille moyenne en effectifs salariés des entreprises défaillantes [Schmitt, 1996]).

La transmission d'entreprises concerne en majorité des entreprises saines pour lesquelles l'offre d'entrepreneurs doit être développée. En 1985, les échecs de transmission ont amputé le tissu économique de 3000 entreprises et entraîné la perte de 27000 emplois. Favoriser la transmission des entreprises semble donc être un impératif, même si le choix des modalités d'intervention est difficile. Privilégier la transmission familiale peut gêner l'ouverture du capital de l'entreprise aux investisseurs externes et en freiner le développement.

En conclusion, la période récente combine des occasions de créations d'entreprises et une offre d'entrepreneurs importante. Les causes expliquant le renouveau entrepreneurial sont diverses et les années 1980 ont permis l'installation d'entrepreneurs :

- développant des innovations dans les niches technologiques de certains secteurs industriels ;

- mettant en œuvre des qualités de manager par les reprises d'entreprises ;

- utilisant un savoir-faire professionnel dans certains secteurs des services aux ménages par le travail à façon ;

- mais aussi profitant des primes proposées par les différentes institutions ${ }^{29}$;

28. Le tribunal a la possibilité de désigner un à cinq contrôleurs parmi les créanciers, sans conditions de montant ou de nature de créance. Ces derniers jouent un rôle d'assistance du représentant des créanciers et du juge commissaire.

29. L'Agence nationale pour la création d'entreprise (ANCE) définit une catégorie d'entrepreneurs dont la motivation première serait de bénéficier du maximum des aides à la création d'entreprises, en particulier des aides régionales qui peuvent atteindre un montant relativement important. Ces entrepreneurs qualifiés de «chasseurs de primes » ont peut-être entraîné une plus grande circonspection dans l'attribution des primes régionales à la création d'entreprises, qui ont disparu de la plupart des politiques d'aides régionales. 
- ou répondant à un impératif économique de survie. Dans ce dernier cas, les entreprises ne peuvent souvent se pérenniser que par une sousrémunération du travail de l'entrepreneur.

Quelle peut être alors l'évolution du système productif dans les années à venir ?

Pour Coase ${ }^{30}$, la dimension de la firme est déterminée par le principe marginaliste, l'entrepreneur recourant au marché à partir du moment où le marché permet de faire mieux que la coordination de l'activité à l'intérieur de l'entreprise. On peut supposer, en suivant la théorie de Coase et les développements de Williamson, que l'essor des nouvelles techniques liées à l'informatique, à la robotique, à l'automatisme, à la communication et à de nouveaux contrats du travail (flexibilité accrue du marché du travail, développement des contrats à durée déterminée, de l'intérim, etc.) vont permettre de rendre le marché plus efficace pour l'entrepreneur et, donc, réduire l'intérêt d'une intégration importante, ce qui diminuera la taille des organisations.

Un nombre élevé de créations d'entreprises individuelles résulte aussi de la volonté des grandes entreprises de contourner le contrat de travail et d'y substituer le contrat commercial plus souple et moins contraignant, et ce plus particulièrement dans certains secteurs (le bâtiment et les travaux publics, les transports routiers, les taxis). Le résultat est alors assez dommageable pour les nouveaux entrepreneurs de ces secteurs, qui voient disparaître une partie de leurs avantages sociaux.

Les moyens mis en œuvre pour favoriser les créations d'entreprises sont divers et il est difficile de privilégier tel ou tel type d'instrument de politique économique puisque la diversité des créateurs d'entreprises, des conditions de création ou des types d'entreprises créées peut justifier différents moyens d'action des pouvoirs publics et locaux. On peut cependant faire quelques constatations :

- les créations d'entreprises dues à la crise répondent en général à un objectif pragmatique de création de sa propre activité;

- les créations d'entreprises dans le secteur des services marchands aux entreprises résultent dans certains cas, de substitutions d'emplois aux réductions d'effectifs des grandes entreprises;

- enfin, les créations d'entreprises innovantes restent par définition les créations privilégiées, celles sur lesquelles, l'organisation territoriale des activités et des réseaux locaux peuvent exercer une influence décisive.

30. R. Coase (1937), «The nature of the firm», Economica. 


\section{Annexe I \\ Les flux d'entreprises}

En France, la source du Système informatique de répertoire des entreprises et des établissements (SIRENE) comptabilise tous secteurs et tous statuts confondus, depuis une quinzaine d'années, entre 210000 et 280000 créations d'entreprises (créations d'entreprises nouvelles et reprises d'entreprises existantes). Ce niveau élevé de renouvellement du tissu productif par rapport à la fin des années 1960 s'est accompagné d'une croissance régulière du nombre des défaillances d'entreprises (de 20895 en 1981 à 63200 en 1993). Celles-ci ont augmenté en raison de la forte mortalité des jeunes entreprises, de l'élargissement des procédures de redressement judiciaire à un nombre croissant d'acteurs économiques et de la faillite employée comme stratégie de gestion dans le cadre d'une législation plutôt favorable à l'entreprise à partir des lois de 1984-1985. Le solde des créations et des disparitions d'entreprises (défaillances et cessations volontaires de l'activité) se traduit cependant par une augmentation du nombre des entreprises.

\section{Annexe II \\ Limites des sources statistiques sur la démographie d'entreprises}

La source de l'Union nationale des entreprises dans l'industrie et le commerce (UNEDIC) représente imparfaitement certains secteurs : exclusion du secteur administratif en totalité, d'une large part du secteur public à caractère industriel et commercial, des secteurs des industries agroalimentaires et des entreprises agri$\operatorname{coles}^{31}$. La source du Système informatique de répertoire des entreprises et des établissements (SIRENE) présente encore des lacunes, lors de l'appariement avec le fichier UNEDIC : en 1990, pour près de $30 \%$ des actifs occupés, l'établissement employeur n'était pas déclaré ou était inconnu dans SIRENE. Les sources sur les flux d'entreprises ne sont pas issues du même fichier que celles sur les stocks (cas des sources du Crédit d'équipement aux petites et moyennes entreprises, CEPME, ou lorsqu'elles le sont (cas du fichier SIRENE), elles présentent des écarts avec l'évolution du stock (absence de prise en compte de l'ensemble des disparitions d'entreprises, en particulier des cessations volontaires d'activité). Par ailleurs, le changement de lieu d'implantation de la production, de statut juridique ou de siège social entraînera des mouvements d'entreprises (disparitions, recréations) qui n'ont pas de signification économique. De même, les fusions apparaissent comme deux fausses disparitions et une apparition (ou fausse création), les fusions-absorptions, comme une fausse disparition et une apparition, l'apport partiel d'actifs, comme une apparition d'entreprise.

31. «L'emploi régional et sectoriel de 1974 à 1991 », Insee Résultats, Emplois-revenus, $\mathrm{n}^{\text {os }}$ 235-236, avril 1993. 


\section{Annexe III \\ L'aide aux chômeurs créateurs ou repreneurs d'entreprises (ACCRE)}

L'aide à la création d'entreprises pour les salariés involontairement privés d'emplois qui créent ou reprennent une entreprise a été proposée à titre expérimental au début de 1977 pour la catégorie des cadres, étendue en août 1977 aux autres salariés «privés involontairement d'emplois » et instituée en 1980. En 1984, le transfert de la charge du dispositif des Associations pour l'emploi dans l'industrie et le commerce (ASSEDIC) à l'État se traduit aussi par l'élargissement des conditions d'attributions, par l'augmentation du montant de l'aide et par l'assouplissement des conditions éventuelles de remboursement en cas de cessation d'activité. Devant le succès obtenu (9000 chômeurs-créateurs d'entreprises aidés en 1979, 29000 , en 1984, et 72000 , en 1986), une réforme issue d'un décret du 26 mars 1987 donne à l'administration la possibilité de contrôler la qualité des projets. Le nombre d'aides distribuées va chuter alors à environ 50000 en 1992, et cela malgré l'extension du dispositif aux bénéficiaires du revenu minimum d'insertion (RMI). La loi quinquennale du 20 décembre 1993 va étendre la mesure aux demandeurs d'emplois non indemnisés inscrits à l'Agence nationale pour l'emploi (ANPE) depuis six mois au moins, et instaure une acceptation tacite de la part de l'administration de l'aide au bout d'un mois, ce qui a pour effet de rendre particulièrement difficile, voire impossible, le contrôle de qualité du projet lancé en 1987. Cette loi, appliquée aux dossiers déposés à compter du 5 avril 1994, fusionne par ailleurs l'ACCRE et le Fonds départemental d'initiative pour les jeunes (FDIJ). Le montant de l'aide est unique et passe à $32000 \mathrm{FF}$; en outre, il n'est plus lié au montant de l'indemnité de chômage perçue par le demandeur. La durée du maintien des droits à la protection sociale gratuite est portée de six à 12 mois.

On assiste à une remontée jusqu'à environ 80000 aides distribuées en 1994 et plus de 50000 pour le premier semestre 1995 . En août 1995, le délai au-delà duquel l'aide est réputée accordée passe d'un à trois mois, et les demandeurs d'emplois indemnisés ne peuvent en bénéficier que s'ils ont été inscrits depuis plus de six mois au cours des 18 derniers mois. La forme de l'aide, le montant et les conditions d'attribution seront dépendants des caractéristiques de l'entreprise créée, et il sera demandé certaines connaissances en gestion au candidat créateurrepreneur. On passe donc réellement d'une aide de droit à une aide où la qualité et la consistance du projet entreront en cause pour son obtention. Plus que le nombre de nouvelles entreprises créées, l'orientation va vers la qualité et les chances de pérennisation de la nouvelle activité, ce qui a entraîné lors de la mise en application de la loi une diminution du nombre des aides distribuées. Effectivement, à partir du milieu de l'année 1995, on enregistre une chute du nombre d'entreprises aidées. Au début de 1997, l'aide a été supprimée. 


\section{Bibliographie}

ALBERT, J. et L. ViALET (1977), «Les défaillances d'entreprises depuis dix ans : un tournant en 1974 », Économie et Statistique, no 95 , décembre, p. 33-41.

ALTMAN, E.I. (1981), «Aggregate influences on business failures rates : a distributed-lag analysis », Revue de l'Association Française de Finance, vol. 2, n 4, p. 243-257.

AMAR, M. et L. CREPON (1990), «Les deux visages de la concentration industrielle: efficacité et rente de situation», Économie et Statistique, n 229, février, p. 5-19.

AshCroft, B., J.H. Love et E. MAlloy (1991), « New firms formation in the British Counties with special reference to Scotland », Regional Studies, vol. 25, p. 395-409.

BALDWIN, J.R. et P.K. GORECKI (1989), « Measuring the dynamics of market structure: concentration and mobility statistics for the Canadian manufacturing sector », Annales d'Économie et de Statistique, $\mathrm{n}^{\text {os }} 15 / 16$, p. 315-332.

BAUMOL, W.J. (1968), «Entrepreneurship in economic theory », American Economic Review, $\mathrm{n}^{0}$ 58, mai.

BerthiER, C. et M.C. PARENT (1994), «Créations, disparitions et restructurations des petites entreprises, les effets sur l'emploi des PME », Économie et Statistique, $\mathrm{n}^{\text {os }} 271 / 272$, p. $13-23$.

BIENAYME, A. (1973), La croissance des entreprises, tome 2, Paris, Bordas.

Blazy, R., P. CHARLETY et J. COMBIER (1993), «Les défaillances d'entreprises, des difficultés visibles plusieurs années à l'avance », Économie et Statistique, $\mathrm{n}^{\text {os }} 268-269$, p. 101-111.

BonNEAU, J. (1996), «Les nouvelles PMI, beaucoup de créateurs malgré les risques », Le 4 pages des statistiques industrielles, SESSI (Services des statistiques industrielles), Ministère de l'Industrie, $\mathrm{n}^{\circ} 58$, février.

BONNEAU, J. et B. THIRION (1997), «Création et devenir des entreprises créées de 1987 à 1995 », Insee Résultats, système productif, $\mathrm{n}^{\text {os }} 125-126-127$, avril.

BONNEAU, J. et D. FRANCOZ (1995a), «Le profil du créateur influence la survie de l'entreprise », Insee Première, $\mathrm{n}^{\circ} 372$, avril, 4 pages.

BONNEAU, J. et D. FRANCOZ (1995b), «L'incidence sur l'emploi des nouvelles entreprises », Insee Première, $\mathrm{n}^{\circ} 415$, novembre, 4 pages.

BONNET, J. (1989), Le renouveau entrepreneurial, Thèse nouveau régime de l'Université de Poitiers, 607 pages.

BonNET, J. (1995), «Les dynamiques régionales et leurs facteurs », Revue d'économie régionale et urbaine, $\mathrm{n}^{\mathrm{0}} 1$, p. 3-34.

BONNET, J. (1997), «Les fortunes diverses du développement régional », Économie et Statistique, septembre-octobre.

Bouba-Olga, O. et B. Guesnier (1995), «Dynamiques des systèmes localisés de production et cadre spatial d'analyse », allocution présentée au colloque Dynamiques industrielles, dynamiques territoriales, Toulouse, 30-31 août et $1^{\text {er }}$ septembre.

BRUNEEL, D. et J.P. PATAT (1983), «Politique du crédit et régulation monétaire », Cahiers Économiques et Monétaires, $\mathrm{n}^{\circ} 18$, novembre-décembre. 
CAILliES, J.M. (1988), «Quatre entreprises sur dix créées depuis 1981 : le tissu productif se renouvelle rapidement », Économie et Statistique, no 206, janvier, p. 3-12.

CAILliES, J.M. (1989), « Être employeur, une décision inscrite dans le projet d'entreprise », Économie et Statistique, $\mathrm{n}^{\circ} 219$, mars, p. 3-10.

Charpail, C. (1995), «L'aide aux chômeurs-créateurs ou repreneurs d'entreprises (ACCRE) en 1994 », Premières synthèses, direction de l'animation de la recherche des études et des statistiques, Dares, $n^{\circ} 106$, août.

COASE, R. (1937), «The nature of the firm», Economica, p. 386-405.

COMBIER, J. (1994), «Les défaillances en 1993 », Insee Première, juillet, 4 pages.

CRUM, W.L. (1953), The Age Structure of the Corporate System, Berkeley, University of California Press.

DE BANDT, J. (1988), «L'économie industrielle face à la réalité des transformations industrielles », dans Le Traité d'Économie Industrielle, sous la direction de R. Arena, L. Benzoni, J. De Bandt, P.M. Romani, Paris, Economica.

DE BARry, C. et B. SAVoye (1994), «Emplois et ventes dans les petites entreprises industrielles pérennes », Économie et Statistique, nos 271-272, p. 37-48.

DELATTRE, M. (1982), «Les PME face aux grandes entreprises », Économie et Statistique, no 148 , octobre, p. 3-20.

DElATtRE, M. (1986), Forces et faiblesses des secteurs industriels 1979-1984, Collection de l'INSEE, série $\mathrm{E}, \mathrm{n}^{\circ} 100$, février.

DEVILLIERS, M. (1987), «Petites, moyennes ou grandes entreprises, sur qui compter?», dans Quel avenir industriel pour la FRANCE, ouvrage présenté par J. Dominati, Paris, Economica, p. 217-233.

DIDIER, M. (1982), « Crise et concentration du secteur productif », Économie et Statistique, $\mathrm{n}^{\mathrm{o}} 144$, mai, p. 3-12.

FernandeZ, A. et P. Pilinski (1993), «L'industrie pharmaceutique, espace national et mondialisation », dans Les nouveaux espaces de l'entreprise, sous la direction de M. Savy et P. Veltz, DATAR, la Tour-d'Aigues, Éditions de l'Aube.

FIZAINE, F. (1968), «Analyse statistique de la croissance des entreprises selon l'âge et la taille», Revue d'Économie Politique, p. 606-620.

FRANCOZ, D. (1996), «Les cessations d'entreprises : méthodes d'estimation et facteurs explicatifs ", série des documents de travail de la direction des statistiques d'entreprises, division administration du répertoire SIRENE et démographie des entreprises, $\mathrm{n}^{\circ}$ 9606, juin.

FRANCOZ, D. et J. BONNEAU (1994), «Le devenir des entreprises créées en 1987 », Insee Première, avril, 4 pages.

GEROSKI, P.A. (1990), «Entry and the dynamics of profit margins », Revue d'Économie Industrielle, $\mathrm{n}^{\circ} 54,4^{\mathrm{e}}$ trimestre, p. 7-21.

GhaZALI, A., B.C. GHOSH et R. TAY (1994), « The determinants of entrepreneurial career choice among university graduates in Singapore », Nanyang Technological University, Singapore, allocution présentée au colloque de l'International Council for Small 
Business (ICSB), Small Business and its Contribution to Regional and International Development, 27-29 juin, Strasbourg.

GIORDANNO, Y. (1987), «Les tendances de la recherche en sciences de gestion depuis 20 ans : le management au centre des préoccupations », Problèmes Économiques, $\mathrm{n}^{\circ}$ 2054, 23 décembre, p. 3-9.

GolleC, M. et P. LAUHLÉ (1987), «La transmission du statut social », Économie et Statistique, $\mathrm{n}^{\text {os }}$ 199-200, mai-juin, p. 85-92.

Grangeas, G. et J.M. LePage (1993), Économie de l'emploi, Paris, Presses universitaires de France, coll. «Économie».

GUESNIER, B. (1994), « Regional variations in new firms formation in France», Regional Studies, vol. 28, p. 347-358.

HARDY, G. (1988), «L'informatisation des PME», Problèmes Économiques, n ${ }^{\circ} 2066$, mars, p. 16-18.

HIGHFIELD, R. et R. SMILEY (1987), « New business starts and economic activity, an empirical investigation », International Journal of Industrial Organisation, vol. 5, p. 51-66.

HounTOUNDJI, G. (1988), « Concurrence sectorielle et démographie des firmes industrielles régionales : une étude économétrique sur la région, Provence-Alpes-Côte-d'Azur », Revue d'Économie Industrielle, $\mathrm{n}^{\mathrm{o}} 45,3^{\mathrm{e}}$ trimestre, p. 59-68.

HounToundJI, G. (1995), «External trade and business failure rates », Document de travail LATAPSES, CNRS, Nice.

Insee Première (1993), «Les petites entreprises innovantes », $\mathrm{n}^{0} 268$, juillet, 4 pages.

Insee Résultats (1993), «L'emploi régional et sectoriel de 1974 à 1991 », Emplois-revenus, $\mathrm{n}^{\text {os }}$ 235-236, avril.

ISARD, W. (1956), Location and Space Economy: A General Theory Relating Industrial Localisation, Market Areas, Land Use, Trade and Urban Structure, New York, M.I.T. et John Wiley \& Sons Inc.

JAYET, H. et A. TORRE (1994), "Vie et mort des entreprises, quelles analyses ? ", Revue d'Économie Industrielle, $3^{\mathrm{e}}$ trimestre, p. 75-92.

Julien, P.A. et M. MARChESNAY (1996), L'entrepreneuriat, Paris, Economica poche.

KEEBLE, D. et S. WALKER (1993), «New firms, small firms and dead firms : spatial patterns and determinants in the United Kingdom », Regional Studies, vol. 28.4, p. 411-427.

KeEble, D. et E. WEWER (1986), New Firms and Regional Development in Europe, Londres, Croom Helm.

KESSIDES, I.N. (1990), «Towards a testable model of entry. A study of the US manufacturing industries », Economica, 57, mai, p. 219-238.

KomвOU, L. (1986), Les créateurs d'entreprises en AQUITAINE : analyse de leurs profils de leurs comportements et de leurs évaluations des aides publiques pendant la période 1977-1984, Thèse d'État en sciences de gestion de l'Université de Bordeaux.

KOMBOU, L. et J. KOCHANSKI (1988), Les créateurs d'entreprises, profils, comportements et conditions de création au cours de la période 1980-1987, Paris, Agence nationale pour la création d'emplois, ANCE. 
LACASSE, R.M. et B. LAMBERT (1988), «La création d'une entreprise francophone minière », Revue Internationale de Gestion des Petites et Moyennes Organisations, vol. 3.

LEIBENSTEIN, H. (1968), «Entrepreneurship and development », American Economic Review, $\mathrm{n}^{\circ} 2$.

Le Monde Initiatives (1995), « Les métamorphoses du travail », publication thématique 17 mai.

Les Notes Bleues de BERCY (1994), « La transmission des PMI à titre gratuit ou onéreux », publication du ministère de l'Économie et du ministère du Budget, 16 au 31 décembre.

MADINIER, H. (1987), «Quelles sont les entreprises qui embauchent?», Le Monde, jeudi 10 septembre.

MARC, F. (1988), « Nouvel entrepreneuriat et mission sociale de l'entreprise», communication présentée au Colloque sur les PME et le développement régional, Montpellier, mai.

MARChESNAY, M. (1984), «La PME dans la crise : panacée ou pis-aller », allocution présentée au Colloque de Trois-Rivières, Les PME et la crise, octobre.

MARCO, L. (1985), «L'analogie biologique et l'économie des faillites », Revue d'Économie Politique, no 1, p. 87-95.

MARCO, L. et M. RAINELli (1986), "Les disparitions de firmes industrielles en France : un modèle économétrique », Revue d'Économie Industrielle, $\mathrm{n}^{\circ}$ 36, $2^{\mathrm{e}}$ trimestre, p. 1-13.

MARCO, L., F. VIEUX et M. DromaIN (1992), «Reprises d'entreprises en Haute-Normandie 1978-1990», Vie et sciences économiques, Revue de l'Andase.

Marshall, A. (1961), Principles of Economics, $1^{\text {re }}$ édition $1890,9^{\mathrm{e}}$ édition, Londres, McMillan.

MAYER, N. (1977), «Une filière de mobilité ouvrière : l'accès à la petite entreprise artisanale et commerciale », Revue Française de Sociologie, $\mathrm{n}^{0}$ XVIII, 1, janvier-mars, p. 25-45.

MONFORT, J.A. et L. VASSILLE (1985), La concentration dans les activités économiques : les établissements, les entreprises, les groupes, Collection de l'INSEE, série E, 98, octobre.

NiJKamP, P. et GiaOuTZI (1988), Informatics and Regional Development, Avebury.

OCDE (1986), Lettre d'information du programme d'action et de coopération concernant les initiatives locales de créations d'emplois (ILE), mars.

OBSERVATOIRE EUROPÉEN DES PME (1995), Troisième rapport annuel, EIM, Small Research and Consultancy, ENSR.

SCHMITT, M.C. (1996), «Les défaillances d'entreprises en FRANCE», Problèmes Économiques, décembre.

SCHWALBACH, J. (1987), «Entry by diversified firms into German industries », International Journal of Industrial Organisation, $\mathrm{n}^{\circ}$ 5, p. 43-50.

SHEPHERD, W.G. (1990), «Mainstream industrial organisation and new schools », Revue Économique, mai, p. 453-480.

VIENNET, H. (1988), «Qui créé une entreprise ou reprend une affaire?», Économie et Statistique, $\mathrm{n}^{\circ} 215$, novembre. 